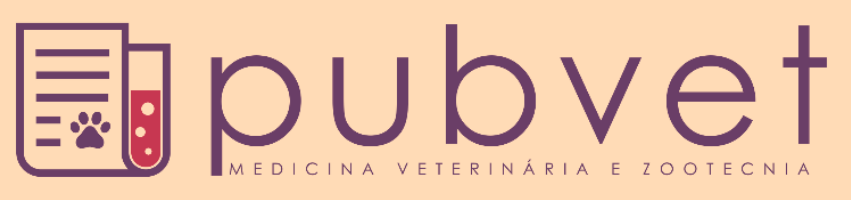

https://doi.org/10.31533/pubvet.v14n8a639.1-5

\title{
Avaliação das principais patologias relacionadas ao uso de contraceptivos em felinos e seus efeitos deletérios
}

\author{
Francisco Lima Silva ${ }^{1 *} \bullet$, Catarina Rafaela Alves da Silva ${ }^{2} \bullet$, Luciana Rangélia Malvina Souza de \\ Castro $^{30}$, Wanderson Gabriel Gomes de Melo $^{30}$, Artur Oliveira Rocha ${ }^{3}$, Aline Carvalho \\ Araújo $^{3}$, Kelline Emanuelle da Rocha Rodrigues ${ }^{4}{ }^{\circ}$, Thanisya Kelly de Paiva Brito ${ }^{4}{ }^{\circ}$, Eglesia \\ Rodrigues Leite Fernandes $^{4}{ }^{\circ}$, Tairine Melo $\operatorname{Costa}^{4}{ }^{\ominus}$, Kelline Emanuelle da Rocha Rodrigues ${ }^{4}$ \\ ${ }^{\text {I}}$ Professor da Universidade Federal do Piauí, Departamento de Clínica e Cirurgia Veterinária. Teresina - PI. Brasil. \\ ${ }^{2}$ Médica veterinária, Anestesiologista Hospital Veterinário Universitário, Universidade Federal do Piauí. Teresina - PI. Brasil. \\ ${ }^{3}$ Graduandos em Medicina Veterinária, Universidade Federal do Piauí. Teresina - PI. Brasil. \\ ${ }^{4}$ Médica veterinária autônoma Teresina - PI. Brasil. \\ *Autor para correspondência, E-mail: flimavet@hotmail.com
}

Resumo. Com a crescente urbanização e individualidade social há o aumento da criação com guarda responsável de animais de estimação, com principais exemplares os cães e gatos, este último como preferencial pelos tutores de forma ascendente nos últimos anos. Naturalmente os felinos podem ter até três gestações no período de um ano, com variação de um a nove filhotes em cada uma delas. Desta forma, a superpopulação destes animais ocorre de maneira exponencial. Esta característica, leva os proprietários a procurar por formas de contracepção para os animais. Dentre as formas existentes, destacam-se a ovariosalpingohisterectomia (OSH) e a contracepção hormonal. Os fármacos anticoncepcionais de maior utilização são os análogos sintéticos da progesterona, como, Acetato de Medroxiprogesterona (AMP), o Acetato de Megestrol e a proligestona. Porém, estes provocam efeitos negativos como hiperplasia endometrial cística, levando à piometra, hiperplasia mamária, resultando em tumores mamários, aborto, diabetes mellitus, dermatopatias e distúrbios comportamentais, portanto não é recomendado o seu uso nesta espécie. Foi realizado um levantamento de dados por meio de questionários, com proprietários de gatas levadas para atendimento clínico do Hospital Veterinário Universitário Jeremias Pereira da Silva da Universidade Federal do Piauí, com o objetivo de analisar quantitativamente os casos de animais com efeitos colaterais, de acordo com a raça e a idade, correlacionado ao uso de fármacos contraceptivos. Os dados obtidos se referem aos meses de março a maio de 2015. Todos os animais descritos neste estudo que apresentaram características patológicas fizeram a utilização de fármacos contraceptivos. Nos 2 casos de piometra encontrados a faixa etária de 4 anos (50\%) e 8 anos $(50 \%)$ foi observada, e todos $(100 \%)$ dos casos ocorreram em gatas Sem Raça definida (SRD). Dos 3 casos de neoplasia mamária, animais com 9 meses correspondem a 33,3\%, 1 ano $(33,33 \%)$ e 2 anos $(33,33 \%)$ de idade, e todos (100\%) eram SRD. Dos 2 casos de abortos, a faixa etária observada foi de 2 anos (50\%) e de 3 anos (50\%) de idade, e as raças afetadas foram SRD (50\%) e siamesa (50\%). Dos 2 casos de hiperplasia mamária observados. em gatas com 6 meses $(50 \%)$ e 8 meses $(50 \%)$ de idade, e a única raça acometida foi a SRD (100\%). Conclui-se que o uso indiscriminado de fármacos contraceptivos ainda é frequente, assim como, é elevada a quantidade de animais que apresentam efeitos negativos ao seu uso.

Palavras chave: aborto, fármacos contraceptivos, hiperplasia mamária, neoplasia mamária, piometra 


\title{
Evaluation of the main pathologies related to contraceptive use in felines and their deleterious effects
}

\begin{abstract}
With the increasing urbanization and social individuality there is the increase of the creation with responsible guarding of pets, with dogs and cats being the main one, the latter being preferred by the tutors of ascending form in the last years. The, felines can have up to three pregnancies within a year, ranging from one to nine pups in each. Thus, the overpopulation of these animals occurs exponentially. This feature leads owners to look for contraception for animals. Among the existing forms, there are ovariosalpingohisterectomy (OSH) and hormonal contraception. The most commonly used contraceptive drugs are synthetic progesterone analogues such as Medroxyprogesterone Acetate (AMP), Megestrol Acetate and proligestone. However, these cause negative effects such as cystic endometrial hyperplasia, leading to pyometra, mammary hyperplasia, resulting in breast tumors, abortion, diabetes mellitus, dermatopathies and behavioral disorders, so their use is not recommended. A survey was carried out through questionnaires, with owners of cats taken for clinical care at the Jeremias Pereira da Silva University Veterinary Hospital of the Federal University of Piauí, aiming to quantitatively analyze the cases of animals with side effects, according to the authors. race and age, correlated with the use of contraceptive drugs. The data obtained refer to the months from March to May 2015. All animals described in this study that presented pathological characteristics made use of contraceptive drugs. In the 2 cases of pyometra found the age range of 4 years (50\%) and 8 years $(50 \%)$ was observed, and all (100\%) cases occurred in cats without defined breed (SRD). Of the 3 cases of breast cancer, animals at 9 months corresponded to $33.3 \%, 1$ year $(33.33 \%)$ and 2 years $(33.33 \%)$ of age, and all (100\%) were SRD. Of the 2 abortion cases, the age range observed was 2 years (50\%) and 3 years $(50 \%)$, and the affected races were SRD $(50 \%)$ and Siamese $(50 \%)$. Of the 2 cases of breast hyperplasia observed. in 6-monthold (50\%) and 8-month-old (50\%) cats, and the only breed affected was the SRD (100\%). It is concluded that the indiscriminate use of contraceptive drugs is still frequent, as well as the number of animals that have negative effects on their use.
\end{abstract}

Keywords: abortion, contraceptive drugs, breast hyperplasia, breast cancer, pyometra

\section{Evaluación de las principales patologías relacionadas con el uso de anticonceptivos en felinos y sus efectos nocivos}

Resumen. Con el aumento de la urbanización y la individualidad social, aumenta la creación con la custodia responsable de las mascotas, siendo los perros y los gatos los principales, siendo estos últimos los preferidos por los tutores de forma ascendente en los últimos años. Naturalmente los felinos pueden tener hasta tres gestaciones en un año, que van de uno a nueve crías en cada una. Por lo tanto, la sobrepoblación de estos animales ocurre exponencialmente. Esta característica lleva a los propietarios a buscar métodos anticonceptivos para los animales. Entre las formas existentes, hay ovariosalpingohisterectomía (OSH) y anticoncepción hormonal. Los anticonceptivos más utilizados son los análogos sintéticos de progesterona, como el acetato de medroxiprogesterona (AMP), el acetato de megestrol y la proligestona. Sin embargo, estos causan efectos negativos como la hiperplasia endometrial quística, que conduce a piometra, hiperplasia mamaria, lo que resulta en tumores de mama, aborto, diabetes mellitus, dermatopatías y trastornos del comportamiento, por lo que no se recomienda su uso. Se realizó una encuesta a través de cuestionarios, con dueños de gatos tomados para atención clínica en el Hospital Veterinario de la Universidad Jeremias Pereira da Silva de la Universidad Federal de Piauí, con el objetivo de analizar cuantitativamente los casos de animales con efectos secundarios, de acuerdo con la raza y edad, correlacionadas con el uso de anticonceptivos. Los datos obtenidos se refieren a los meses de marzo a mayo de 2015. Todos los animales descritos en este estudio que 
presentaron características patológicas utilizaron medicamentos anticonceptivos. En los 2 casos de piometra se observó un rango de edad de 4 años (50\%) y 8 años (50\%), y todos los casos (100\%) ocurrieron en gatas sin raza definida (SRD). De los 3 casos de cáncer de mama, los animales con 9 meses correspondían al 33,3\%, 1 año $(33,33 \%)$ y 2 años $(33,33 \%)$ de edad, y todos (100\%) eran SRD. De los 2 casos de aborto, el rango de edad observado fue de 2 años (50\%) y 3 años (50\%), y las razas afectadas fueron SRD $(50 \%)$ y siamesas (50\%). De los 2 casos de hiperplasia mamaria observados, en gatos con 6 meses (50\%) y con 8 meses (50\%), y la única raza afectada fue la SRD (100\%). Se concluye que el uso indiscriminado de medicamentos anticonceptivos sigue siendo frecuente, así como la cantidad de animales que tienen efectos negativos en su uso.

Palabras clave: aborto, anticonceptivos, hiperplasia mamaria, cáncer de mama, piometra

\section{Introdução}

A urbanização cada vez mais crescente vem trazendo hábitos coletivos entre os indivíduos, que isolados em seus lares, acabam criando fortes laços afetivos com os animais, sendo os felinos uma das espécies em maior expansão (Santana \& Oliveira, 2006). A guarda responsável de animais de estimação pode ser caracterizada por um conjunto de ações que visam promover a saúde e o bemestar animal, além da preservação do meio ambiente. No Brasil, existem legislações vigentes relacionadas à regulamentação da guarda e controle da população de animais (Balizardo, 2015).

Em condições naturais, gatas podem produzir duas a três ninhadas de um a nove filhotes por ano, gerando problemas de superpopulação, levando os proprietários a procurar por métodos anticoncepcionais (Domingos et al., 2007). Entre as opções disponíveis para prevenção da gestação em gatas, destacam-se a OSH e a contracepção hormonal. A contracepção hormonal pode causar efeitos colaterais como aumento de peso, hiperplasia ou neoplasia mamária, piometra, diabetes mellitus e supressão adrenal (Filgueira et al., 2008; Viana et al., 2012).

Devido à grande casuística observada de gatas atendidas apresentando efeitos adversos ao uso de fármacos contraceptivos, que são obtidos sem nenhuma orientação médica e falta de regulamentação de controle à sua comercialização, esse estudo foi realizado com o objetivo de analisar quantitativamente os casos de efeitos colaterais, de acordo com a raça, a idade e o uso de fármacos contraceptivos, podendo assim observar a relação entre os pacientes que apresentam patologias com o uso de tais fármacos.

\section{Material e métodos}

É O presente estudo foi desenvolvido no Hospital Veterinário Universitário Médico Veterinário Jeremias Pereira da Silva, Campus Ministro Petrônio Portela, Centro de Ciências Agrárias, da Universidade Federal do Piauí. Os dados obtidos referem-se aos meses de março a maio de 2015, com o total de três meses, nos quais foram avaliados 21 animais da espécie felinas, todos levados para atendimento clínico veterinário no hospital.

Os dados a respeito das fêmeas felinas foram coletados a partir de um questionário previamente elaborado contendo, a identificação do animal, por meio de nome, raça e idade. E posteriormente o questionamento acerca do uso ou não de fármacos anticoncepcionais e da observação de efeitos colaterais.

\section{Resultados e discussão}

No questionário aplicado verificou-se que $52,4 \%$ dos proprietários relataram nunca haver utilizado anticoncepcional em seus animais (Figura 1). Todos os animais eram SRD e tinham entre 6 meses e 8 anos. Dentre as patologias relatadas, $22,2 \%$ dos casos foram de piometra, 22,2\% de aborto e 55,5\% de alterações mamárias (hiperplasia ou neoplasia) (Figura 2). $100 \%$ dos animais que apresentaram estas patologias fizeram uso de anticoncepcionais.

Montanha et al. (2012) e Dias et al. (2013) afirmam que o uso inadequado de fármacos contraceptivos para evitar gestações indesejadas em felinos podem resultar em hiperplasia endometrial cística com infecção de útero, sendo mais comum em gatas com mais de 4 anos 
corroborando com os dados encontrados, nos quais $50 \%$ das gatas com piometra tinham 4 anos, e $50 \%$ tinham 8 anos.

\section{Uso de anticoncepcionais em felinas}

- Nunca utilizaram Já utilizaram

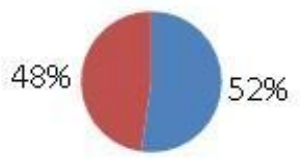

Figura 1. Relato do uso ou não de anticoncepcionais em felinas através de questionários aplicados a proprietários no Hospital Veterinário Jeremias Pereira da Silva da Universidade Federal do Piauí.

O aborto, segundo Montanha et al. (2012) é uma das consequências mais comuns dos fármacos anticoncepcionais, por causar alterações no relaxamento da cérvix. Togni et al. (2018) citam haver forte correlação entre o uso de progestágenos sintéticos e o desenvolvimento de tumorações benignas e malignas nas mamas de felinos, o presente trabalho corrobora tal correlação, ao ser observado grande proporção de casos de neoplasia mamária mediante uso de anticoncepcionais. A literatura cita que as neoplasias mamárias ocorrem em todas as idades, sendo observado em animais entre 9 meses e 2 anos de idade no presente estudo.

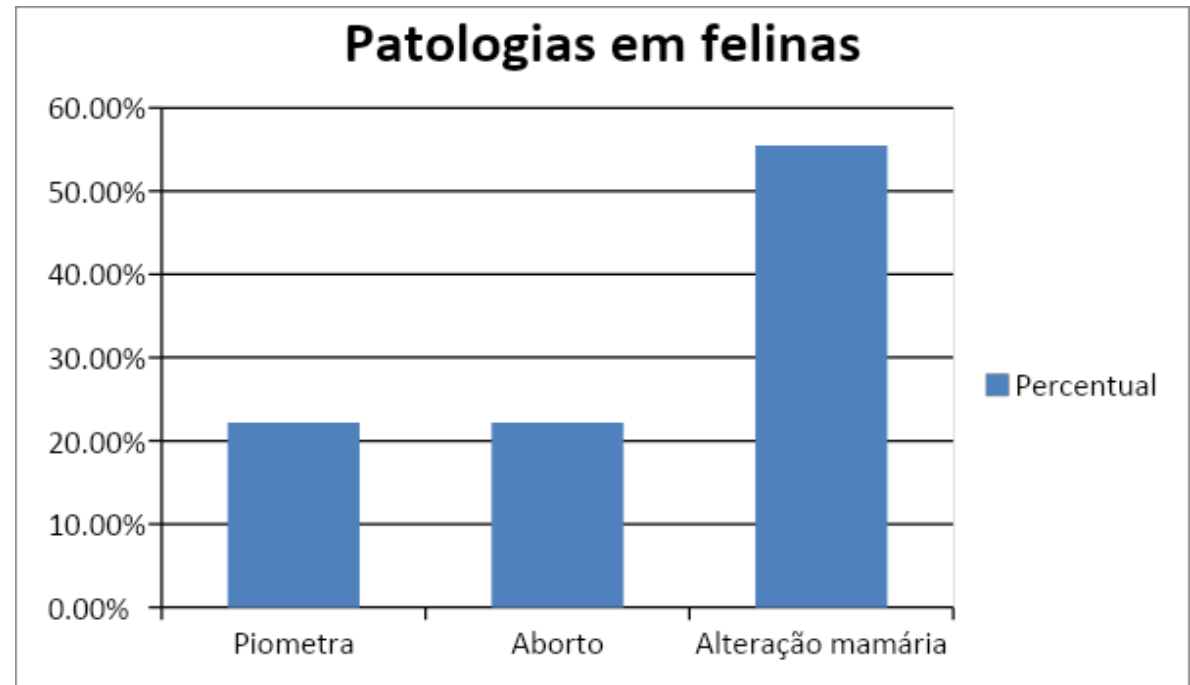

Figura 2. Frequência das principais patologias reprodutivas diagnosticadas em gatas atendidas no Hospital Veterinário Jeremias Pereira da Silva da Universidade Federal do Piauí.

\section{Conclusão}

Pode-se concluir neste estudo que o uso indiscriminado de fármacos contraceptivos ainda é frequente, assim como, é elevada a quantidade de animais que apresentam efeitos negativos decorrentes do seu uso. Este estudo mostrou resultados relacionados ao uso de medicamentos contraceptivos, patologias associadas ao seu uso e a relação entre idade e raças concordando e contribuindo com outros trabalhos já realizados.

A real conscientização dos proprietários de animais frente aos riscos oferecidos pelos fármacos contraceptivos para a saúde e bem-estar dos animais é fundamental, para assim, minimizar o uso e consequentemente os efeitos deletérios nos animais de companhia. 
É necessário que os médicos veterinários a partir da primeira consulta do animal, já informem ao proprietário a melhor forma de prevenir uma gestação, e os efeitos negativos que os fármacos anticoncepcionais podem causar. Deve-se incentivar, mostrando que a castração é o método contraceptivo mais eficaz para o animal, enfatizando as patologias que podem estar sendo prevenidas como também as proles indesejadas.

\section{Referências bibliográficas}

Balizardo, E. (2015). Cartilha de defesa animal. MPSP.

Dias, L. G. G. G., Oliveira, M. E., Dias, F. G. G., Calazans, S. G., \& Confort, V. A. (2013). Uso de fármacos contraceptivos e seu efeitos adversos em pequenos animais. Enciclopédia Biosfera, 9(16), 2077.

Domingos, I. H., Rigo, L., \& Honer, M. R. (2007). Perfil das populações canina e felina no município de Campo Grande, MS. Ensaios e Ciência: Ciências Biológicas, Agrárias e Da Saúde, 11(1), 97-103.

Filgueira, K. D., Costa Paulo Fernando Cisneiros, R., \& Paula, V. V. (2008). Hiperplasia mamária felina: sucesso terapêutico com o uso do aglepristone. Ciência Animal Brasileira, 9(4), 1010-1016.

Montanha, F. P., Corrêa, C. S. de S., \& Parra, T. C. (2012). Maceração fetal em gata em decorrência do uso de contraceptivos - Relato de caso. Revista Científca Eletrônica de Medicina Veterinária, 10(19), 1-6.

Santana, L. R., \& Oliveira, T. P. (2006). Guarda responsável e dignidade dos animais. Revista Brasileira de Direito Animal, 1, 207-230.

Togni, M., Curtis, A., Vargas, D. P., Kommers, G. D., Irigoyen, L. F., \& Fighera, R. A. (2018). Causas de morte e razões para eutanásia em gatos na Região Central do Rio Grande do Sul (1964-2013). Pesquisa Veterinária Brasileira, 38(4), 741-750. DOI: https://doi.org/10.1590/1678-5150-pvb-5075

Viana, D. C., Santos, A. C., Rui, L. A., Oliveira, D. M., Silva, A. B., Costa, F. das C. F. C., \& Assís Neto, A. C. (2012). Hiperplasia mamária felina: Um relato de caso. Veterinária Notícias, 18(2).

Licenciamento: Este artigo é publicado na modalidade Acesso Aberto sob a licença Creative Commons Atribuição 4.0 (CC-BY 4.0), a qual permite uso irrestrito, distribuição, reprodução em qualquer meio, desde que o autor e a fonte sejam devidamente creditados. 